\title{
Cribado de infección por citomegalovirus congénito en recién nacidos de alto riesgo
}

\author{
Congenital cytomegalovirus infection screening in high risk newborn
}

\author{
Ángela Chuang Chuang1, Hernán Ramos Hernández²,3, Úrsula Zelada Bacigualupo 1,4, María Teresa López Castillo5,6, Leonel Villavicencio \\ Landeros7 , Luisa Montecinos Peret 7, Claudio González Muñoz ${ }^{8}$, Tamara Barría Espinoza9 y Giannina Izquierdo Copiz'5, 10
}

1Servicio de Otorrinolaringología, Complejo Asistencial Barros Luco Trudeau, Santiago.
2Servicio de Fonoaudiología, Complejo Asistencial Barros Luco Trudeau, Santiago.
${ }^{3}$ Escuela de Fonoaudiología, Facultad de Ciencias de la Salud, Universidad San Sebastián, Santiago.
${ }^{4}$ Departamento de Otorrinolaringología, Facultad de Medicina, Universidad de Chile.
${ }^{5}$ Servicio de Neonatología, Complejo Asistencial Barros Luco Trudeau, Santiago.
${ }^{6}$ Departamento de Pediatría y Cirugía Infantil Sur, Facultad de Medicina Universidad de Chile.
${ }^{7}$ Laboratorio de Biología Molecular, Hospital Dr. Lucio Córdova, Santiago.
${ }^{8}$ Unidad de Farmacia Clínica, Hospital de Niños Dr. Exequiel González Cortés, Santiago.
${ }^{9}$ Servicio de Otorrinolaringología, Hospital Del Salvador, Santiago.
${ }^{10}$ Unidad de Infectología, Hospital de Niños Dr. Exequiel González Cortés, Santiago.
Los autores no refieren conflictos de interés.
Estudio financiado parcialmente por fondos del Concurso de Proyectos de Investigación de Laboratorio SAVAL-2017 para compra de reactivos de determinación de CMV.

Recibido: 20 de octubre de 2020 / Aceptado: 22 de diciembre de 2020

\section{Resumen}

Introducción: La infección congénita por citomegalovirus $(\mathrm{CMVc})$ es la causa más frecuente de infección intrauterina, $90 \%$ de los recién nacidos (RN) son asintomáticos al nacer y 6 a $15 \%$ desarrollarán secuelas a largo plazo, siendo la principal etiología de hipoacusia sensorio-neural no-genética. Objetivo: Determinar la prevalencia de CMVc en RN de alto riesgo. Pacientes y Método: Estudio de cohorte prospectivo, incluyó $\mathrm{RN}$ hospitalizados, con uno o más de los siguientes criterios: peso de nacimiento $<1.500 \mathrm{~g},<32$ semanas edad gestacional (EG), pequeños para edad gestacional (PEG) severos, sospecha de infección congénita o que "no pasan" en estudio auditivo al nacer, además de hijos de madre con infección por VIH. Se realizó reacción de polimerasa en cadena para CMV en orina antes de 21 días de vida. Resultados: Se enrolaron 193 RN. Prevalencia global CMVc 2,6\% (n: 5) y por grupo de riesgo: 1/3 (n: 1) en RN con sospecha activa de infección congénita, $8,3 \%$ en RN con resultado "no pasa" en estudio auditivo, $4,9 \%$ en hijos de madre con infección por VIH, 3,3\% en PEG severo y $1,7 \%<1500 \mathrm{~g}$, ninguno con asociación significativa. Sólo un paciente con CMVc fue sintomático, quien falleció en el período neonatal y los restantes $\mathrm{RN}$ con $\mathrm{CMVc}$ (asintomáticos) tienen seguimiento auditivo normal. Discusión: La prevalencia reportada es comparable a las internacionales. Recomendamos cribado de CMVc, al menos en grupos de riesgo, siendo lo ideal el cribado universal. Esto permitiría su tratamiento oportuno y un seguimiento activo.

Palabras clave: prevalencia; cribado neonatal; citomegalovirus; hipoacusia sensorio-neural.

\section{Abstract}

Background: Congenital cytomegalovirus infection (cCMV) is the most frequent cause of congenital infection, $90 \%$ of affected newborn (NB) are asymptomatic at birth and $6-15 \%$ will develop long term sequalae. It is the main etiology of non-genetic sensorineural hearing loss. Aim: To determine prevalence of CMV in high risk NB. Methods: Cohort prospective study, including inpatient NB with one or more of following criteria: birth weight $<1,500 \mathrm{~g},<32$ weeks gestational age (GA), severe small for gestational age (SGA), suspected congenital infection or "refer" in newborn hearing test, also NB to HIV-infected mothers. Urine CMV polymerase chain reaction was performed within 21 day of life. Results: 193 NB were enrolled. Global cCMV prevalence $2.6 \%$ (n: 5) and by risk group: one third (n: 1) in NB with suspected congenital infection, $8.3 \%$ in NB with "refer" result in hearing test, $4.9 \%$ in NB to HIV-infected mothers, $3.3 \%$ in severe SGA and $1.7 \%$ in $<1,500 \mathrm{~g}$, none with significant association. Only one symptomatic cCMV was detected who died in neonatal period and the remaining (asymptomatic) cCMV patients have normal hearing follow-up. Discussion: Reported prevalence was comparable to international reports. We recommend cCMV screening, at least in risk groups, being ideal the universal screening. This would allow timely treatment and active follow-up.

Keywords: prevalence; newborn screening; cytomegalovirus; sensorineural hearing loss. 


\section{Introducción}

$\mathrm{E}$ 1 citomegalovirus (CMV) es la causa más frecuente de infección congénita, con prevalencias que varían según región del mundo y nivel socio-económico, siendo en general entre 0,2 y $2,4 \%{ }^{1-7}$ para los recién nacidos $(\mathrm{RN})$ vivos. La infección fetal puede ocurrir por una primoinfección materna durante el embarazo (riesgo de transmisión de 30 a $40 \%$ en madres que eran seronegativas antes de embarazarse) o secundaria a una reactivación o reinfección viral (infección no primaria) (riesgo de 1 a $2 \%$ en madres seropositivas previo al embarazo ${ }^{8}$ y el diagnóstico se realiza con la detección de material genético en orina antes de los 21 días de vida (ddv) ${ }^{9}$. El $90 \%$ de los RN con infección congénita por CMV (CMVc) son asintomáticos al nacer y entre 6 y $15 \%{ }^{10}$ de ellos desarrollarán secuelas a largo plazo como alteraciones del neurodesarrollo o hipoacusia sensorio-neural (HSN). El CMVc es la principal etiología de HSN no genética, pudiéndose atribuir a esta infección entre 6 y $30 \%$ de ellas ${ }^{11}$, a $34 \%$ de las hipoacusias tardías de origen desconocido y hasta un quinto de las HSN profundas bilaterales ${ }^{1,2}$. De los pacientes que desarrollarán HSN por CMVc, sólo $50 \%$ será detectable en el período neonatal a través de programas de Tamizaje Auditivo Universal (TAU) ${ }^{1,2}$

El tratamiento de la infección se reserva, en la actualidad, para aquellos pacientes con compromiso de sistema nervioso central o enfermedad de órgano blanco (sintomáticos), entre los que se incluye la $\mathrm{HSN}^{12}$. Se ha determinado que con el tratamiento antiviral se puede prevenir, e incluso mejorar, el deterioro auditivo si es iniciado antes del mes de vida $a^{4,9,13}$.

En Chile la tasa de infección congénita por CMV fue reportada por Stagno y cols. ${ }^{6}$ y Luchsinger y cols., ${ }^{14} \mathrm{en}$ la década de los $80-90$, con prevalencias de 1,7 y $1,8 \%$, respectivamente, no existiendo nuevos registros a la fecha de esta comunicación. Si bien la prevalencia de CMVc es mayor que la de otras enfermedades de cribado neonatal universal (como la fenilcetonuria y el hipotiroidismo congénito $)^{15}$, su incorporación a dichos programas es aún discutida internacionalmente ${ }^{1,16-18}$. La estrategia de cribado universal neonatal de CMV permitiría identificar en forma temprana la infección en pacientes asintomáticos con riesgo de desarrollar HSN de inicio tardío, y realizar un seguimiento auditivo hasta la edad escolar, de manera de intervenir en forma oportuna y mejorar el pronóstico ${ }^{17,18}$.

Otra estrategia de cribado descrita, es la realizada en grupos de riesgo o "targeted screening", en que se identifican a RN con mayor probabilidad de presentar CMVc; como aquellos que "refieren" o "no pasan" en el estudio auditivo neonatal ${ }^{2}$, donde se estima una frecuencia de infección por CMV entre 1 y $6 \% \%^{2,4,11,19}$. Otros grupos de
$\mathrm{RN}$ con mayor riesgo de CMVc son los hijos de madre con infección por VIH (por presentar éstas un mayor riesgo de reactivación/reinfección durante el embarazo) y los prematuros extremos, con una prevalencia de infección de $4,5^{20}$ y $2,3 \%{ }^{21,22}$, respectivamente. Se estima que un tercio de las infecciones por CMVc cursan con parto prematuro y la mitad de estos $\mathrm{RN}$ son pequeños para la edad gestacional (PEG) ${ }^{23}$. A su vez, quienes nacen con menos de 32 semanas de edad gestacional (EG) y menos de $1.500 \mathrm{~g}$ de peso $^{12}$, presentan un mayor riesgo de adquirir la infección de forma post-natal (CMVp) definida como la detección de CMV en orina después de la tercera semana de vida. El cribado al nacimiento en este grupo permitiría detectar la infección congénita y, a su vez, realizar el diagnóstico diferencial adecuado de $\mathrm{CMVp}^{24}$.

En nuestro país no existen políticas de detección universal de CMV en $\mathrm{RN}$ y el estudio auditivo neonatal hasta ahora sólo está garantizado para los $\mathrm{RN}<32$ semanas EG y/o $<1.500 \mathrm{~g}$ al nacer. Sin embargo, recientemente se aprobó la incorporación del estudio auditivo neonatal al programa público de salud de atención del parto, por lo que se espera la rápida masificación de los programas de $\mathrm{TAU}^{25}$.

El objetivo de este estudio fue determinar la prevalencia de infección por CMV en RN de alto riesgo del Complejo Asistencial Barros Luco Trudeau (CABL).

\section{Pacientes y Método}

Estudio de cohorte, prospectivo, de cribado de CMV en orina a RN de alto riesgo del Servicio de NeonatologíaPuerperio del CABL entre el 1 de julio 2018 y 30 de abril 2019. Se incluyó a todos los RN hospitalizados de alto riesgo, definidos con uno o más de los siguientes criterios: peso de nacimiento $(\mathrm{PN})<1.500 \mathrm{~g}, \mathrm{EG}<32$ semanas, PEG severo (menor a percentil 3 de peso para la EG), aquellos que fueron "referidos" por estudio auditivo neonatal en su primera etapa, y RN con sospecha de infección congénita por signos clínicos, alteraciones sugerentes en la ecografía prenatal o alteración de exámenes de laboratorio al nacer. También se incluyeron los hijos de madre con infección por VIH (hospitalizados en neonatología o en puerperio) nacidos en este período. Se excluyeron del estudio a RN cuyos padres o tutores legales rechazaron la participación, RN sin estudio de CMV antes de los 21 ddv, fallecimiento previo a la obtención de muestra para reacción de polimerasa en cadena (RPC) de CMV y RN con malformaciones o síndromes asociados a hipoacusia.

De acuerdo con el programa de TAU establecido en nuestro centro, todos los RN son estudiados previo a su egreso o días después del alta (en caso de alta en fines de semana). Los RN hospitalizados o con factores de riesgo de hipoacusia ${ }^{26}$ se les realiza potenciales evocados audi- 
de CMV). Las variables continuas fueron expresadas como promedio (desviación estándar) o mediana (rango intercuartil-RIQ o rango mínimo-máximo), según su distribución normal; para evaluar su diferencia entre grupos se utilizó la prueba de $\mathrm{T}$ de Student para las variables con distribución normal y la prueba de la U de Mann-Whitney para las no normales. Las variables categóricas fueron expresadas como porcentaje (frecuencia) y se utilizó la prueba exacta de Fischer para determinar diferencias significativas entre grupos. Se consideró una diferencia significativa si el valor $\mathrm{p}$ del contraste de hipótesis fue menor a 0,05 . Para el análisis estadístico se utilizó el programa STATA 13.

\section{Resultados}

Durante el período de estudio nació un total de 3.718 $\mathrm{RN}$ vivos en el CABL; 246 (6,6\%) de los $\mathrm{RN}$ cumplieron criterios de inclusión, siendo reclutados un total de 193 RN (Figura 1). Los RN excluidos fueron 53 (21,5\%): en $41 \mathrm{RN}$ no se logró realizar estudio de CMV oportunafísico completo, hemograma, pruebas de función hepática (transaminasas y bilirrubinemia), función renal (creatininemia y nitrógeno ureico), RPC para CMV en sangre, estudio de líquido cefalorraquídeo (LCR) (citoquímico y RPC-CMV en LCR), fondo de ojo, ecografía cerebral (USc) y estudio auditivo mediante PEAT automatizado.

Se definió como RN con CMVc sintomático a aquel con alteración significativa del examen físico (púrpura, hepato-esplenomegalia, ictericia, hipotonía, microcefalia), trombocitopenia $<100.000$ plaquetas $/ \mathrm{mm}^{3}$, GPT $>80 \mathrm{IU} / \mathrm{L}$, hiperbilirrubinemia directa $>2 \mathrm{mg} / \mathrm{dL}$, corioretinitis, alteraciones significativas en las neuroimágenes (USc o resonancia magnética) o hipoacusia diagnosticada por PEAT clínico.

Se confeccionó una base de datos anónima con variables seleccionadas para su posterior análisis. Las variables analizadas fueron: sexo, EG, PN, talla nacimiento, circunferencia craneal (CC), edad materna, paridad, nacionalidad materna, resultado auditivo y otros factores de riesgo de hipoacusia.

Todos los RN con infección congénita fueron seguidos en la Unidad de Infectología del Hospital de Niños Dr. Exequiel González Cortés y en el Servicio de Otorrinolaringología del CABL.

El estudio fue aprobado por el Comité de Ética del Servicio de Salud Metropolitano Sur y se solicitó consentimiento informado a las madres/padres o tutores legales de cada RN.

\section{Análisis estadístico}

El tamaño muestral fue por conveniencia (limitado por los recursos disponibles para reactivos de determinación

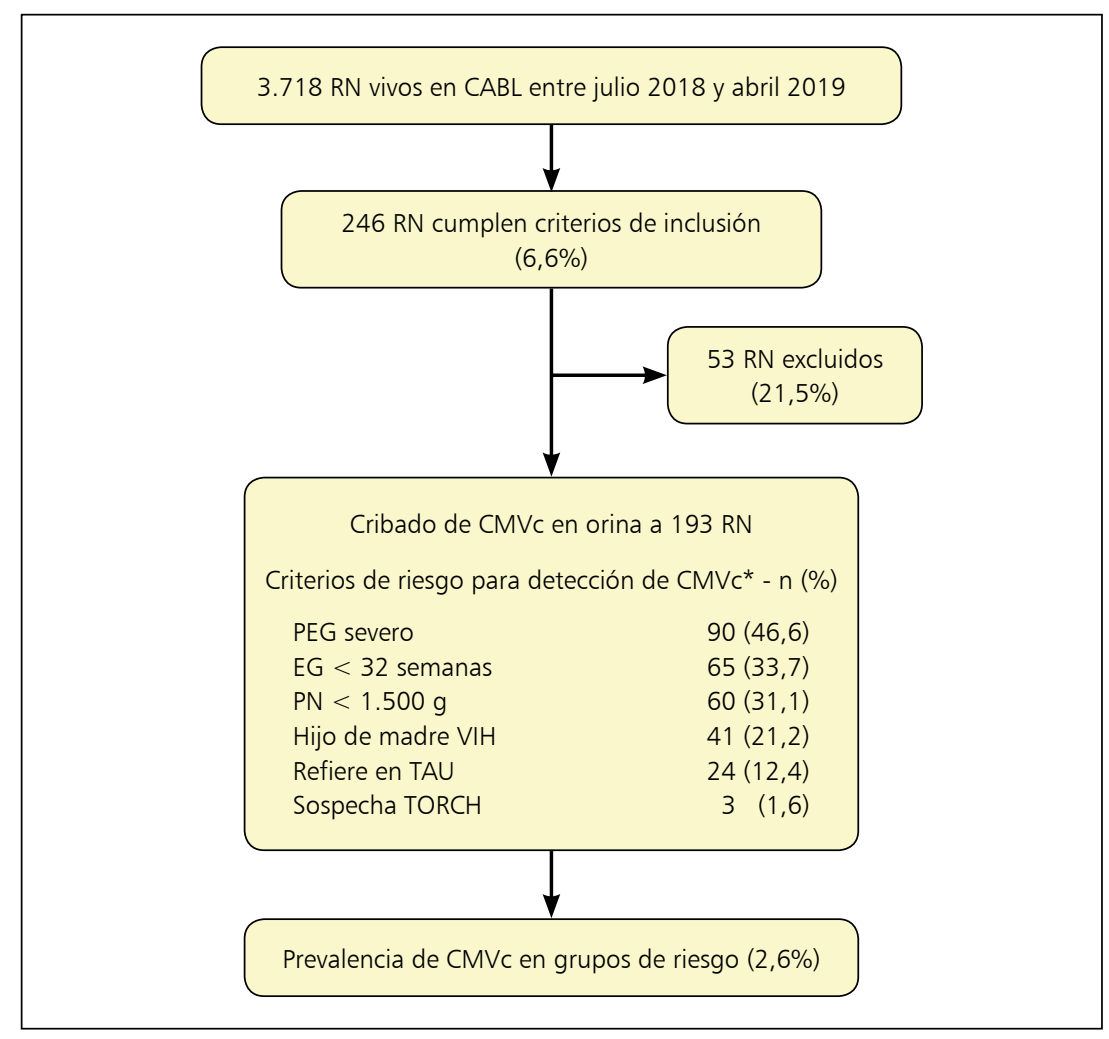

Figura 1. Flujograma de selección de recién nacidos para el cribado en orina de CMV. *Acepta más de un criterio de ingreso. RN: recién nacidos. CABL: complejo asistencial Barros Luco. CMVc: citomegalovirus congénito. PEG: pequeño para la edad gestacional. EG: edad gestacional. PN: peso de nacimiento. VIH: virus de la inmunodeficiencia humana. TAU: tamizaje auditivo universal. TORCH: infección congénita. 
mente (por alta durante fin de semana [fuera de horario de reclutamiento para el estudio], traslado a otro centro o rechazo de participación), $8 \mathrm{RN}$ por fallecimiento previo al estudio de RPC-CMV (prematuros extremos con mediana PN 712 g y EG 24 semanas) y 4 RN por síndrome o malformación asociada a hipoacusia (síndrome CHARGE y microtia).

Del total (Tabla 1), 54\% $(\mathrm{n}=104)$ eran de sexo femenino, con una mediana de EG de 35 (RIQ 30-37) semanas y de PN de 1.880 g (RIQ 1.336-2.525), 42,5\% $(\mathrm{n}=82)$ son hijos de madre inmigrante, con $33,7 \%$ de origen haitiano.

El rango de toma de muestra para RPC-CMV fue de 0 a $21 \mathrm{ddv}$, con mediana de $5 \mathrm{ddv}$.

No se encontraron diferencias estadísticamente significativas entre el grupo de $\mathrm{RN}$ con y sin infección por $\mathrm{CMVc}$ en EG, PN, talla, CC y nacionalidad materna. En el grupo de infección por CMVc se observó una mayor proporción de RN con PEG severo $(n=3 / 5)$ y con estudio auditivo neonatal con resultado "refiere" $(n=2 / 5)$; sin embargo, no fueron diferencias estadísticamente significativas ( $\mathrm{p}=0,44$ y $\mathrm{p}=0,12$, respectivamente) (Tabla 1$)$.

Cinco RN presentaron infección por $\mathrm{CMV}$, con una prevalencia global de CMVc de 2,6\% (IC 95\% - 1 a 6\%); sus características se describen en la Tabla 2. Cuatro de ellos son hijos de madre primigesta, con una mediana de edad de 23 años (rango mínimo-máximo 20-29) y tres de ellas inmigrantes. El diagnóstico positivo de infección por CMVc se realizó con una mediana de $1 \mathrm{ddv}$ (rango mínimo-máximo 0-6). Cuatro pacientes se catalogaron como asintomáticos después de la evaluación inicial, con una mediana de PN 1.865 g (rango mínimo-máximo 1.400-3.506) y EG de 38 semanas (rango mínimo-máximo 35-38). Los casos 1 y 3 , fueron pesquisados por el criterio de PEG severo, sin otro hallazgo significativo y otros dos por el antecedente de ser hijos de madre con infección por VIH.

El único paciente sintomático (caso 4) fue un RN de pretérmino de 36 semanas, adecuado para la EG, quien

\begin{tabular}{|c|c|c|c|c|c|}
\hline & & $\begin{array}{l}\text { Total de RN con cribado CMVc } \\
\qquad n=193\end{array}$ & $\begin{array}{c}\text { CMVc positivo } \\
n=5\end{array}$ & $\begin{array}{l}\text { CMVc negativo } \\
n=188\end{array}$ & Valor $\mathbf{p}^{1}$ \\
\hline Edad gestacional (semanas) & Mediana (RIQ) & $35(30-37)$ & $38(36-38)$ & $35(29-37)$ & 0,05 \\
\hline Peso nacimiento (g) & Mediana (RIQ) & $1880(1336-2525)$ & $2390(1970-3410)$ & $1850(1308-2500)$ & 0,11 \\
\hline Talla (cm) & Mediana (RIQ) & $42(38-46)$ & $45(44-47)$ & $42(38-46)$ & 0,06 \\
\hline Circunferencia craneal (cm) & Mediana (RIQ) & $30.5(27,5-33)$ & $32(30-35)$ & $30,5(27,5-33)$ & 0,18 \\
\hline Prematuros extremos ${ }^{2}$ & n (\%) & $48(24,8)$ & $0(0)$ & $51(27,1)$ & 0,21 \\
\hline PEG severo ${ }^{3}$ & $\mathrm{n}(\%)$ & $90(46,6)$ & $3(60)$ & $87(46,5)$ & 0,44 \\
\hline Refieren tamizaje auditivo ${ }^{4}$ & $\mathrm{n}(\%)$ & $24(12,4)$ & $2(40)$ & $22(11,7)$ & 0,12 \\
\hline
\end{tabular}

\begin{tabular}{|c|c|c|c|c|c|c|c|c|c|c|}
\hline Caso & Sexo & PN (g) & $\begin{array}{l}\text { Talla } \\
(\mathrm{cm})\end{array}$ & $\begin{array}{c}\text { EG } \\
\text { (sem) }\end{array}$ & $\begin{array}{l}\mathrm{CC} \\
(\mathrm{cm})\end{array}$ & $\begin{array}{c}\text { Criterio } \\
\text { inclusión }\end{array}$ & $\begin{array}{l}\text { Edad al Dg } \\
\quad(d d v)\end{array}$ & $\begin{array}{l}\text { Síntomas } \\
\text { CMV }\end{array}$ & Tto CMV & $\begin{array}{c}\text { Seguimiento auditivo a } 12 \\
\text { meses/otros }\end{array}$ \\
\hline 1 & M & 1.400 & 43 & 35 & 29,5 & $\begin{array}{c}\text { PN } \\
\text { PEG severo }\end{array}$ & 6 & No & No & Normoacusia \\
\hline 2 & M & 3.506 & 47 & 38 & 35 & $\begin{array}{l}\text { VIH materno } \\
\text { PEAT }\end{array}$ & 1 & No & No & $\begin{array}{l}\text { Normoacusia } \\
\text { VIH positivo }\end{array}$ \\
\hline 3 & M & 1.970 & 44 & 38 & 30 & PEG severo & 1 & No & No & Normoacusia \\
\hline 4 & M & 3.410 & 49 & 36 & 40 & PEAT, TORCH & 7 & $\mathrm{Si}$ & $\mathrm{Si}$ & Refiere bilateral (neonatal) - Fallece \\
\hline 5 & M & 2.390 & 45 & 38 & 32 & $\begin{array}{l}\text { VIH materno } \\
\text { PEG severo }\end{array}$ & 1 & No & No & Normoacusia \\
\hline
\end{tabular}


presentó hepato-esplenomegalia y petequias al nacer, por lo que se sospechó activamente una infección congénita. Recibió tratamiento con ganciclovir endovenoso; sin embargo, falleció a los $19 \mathrm{ddv}$ por falla multiorgánica. Los cuatro pacientes asintomáticos, al momento de la publicación, se encuentran con normoacusia con un seguimiento de al menos 12 meses en todos ellos.

En la Tabla 3 se describe la prevalencia de infección por CMVc según los grupos de riesgo definidos. Uno de los tres pacientes con sospecha activa de infección congénita presentó RPC-CMV en orina positiva antes de los 21 ddv. Los hallazgos ecográficos o clínicos de sospecha fueron: agenesia de cuerpo calloso y ventriculomegalia supratentorial a la USc en el primer caso, un segundo caso de ventriculomegalia unilateral y un tercero (el único con infección sintomática por $\mathrm{CMVc}$ ) que presentó hepatoesplenomegalia, púrpura y trombocitopenia al nacer. Le siguen en frecuencia los RN que fueron incluidos por estudio auditivo con resultado "refiere" $(8,3 \%)$, los hijos de madre con infección por VIH (4,9\%) y los PEG severos $(3,3 \%)$. No se encontraron diferencias significativas entre los grupos; los pacientes pertenecientes a dos o más grupos de riesgo presentaron $43 \%$ más de prevalencia de CMVc que los pertenecientes a un solo grupo de riesgo, aunque esta diferencia no fue significativa (diferencia porcentual 1\%; IC 95\% 4,1-6,2\%).

En la primera etapa del estudio auditivo neonatal 24/193 (12,4\%) RN fueron "referidos". Dos pacientes del grupo con CMVc: uno con re-evaluación auditiva dando normoacusia y el otro CMVc sintomático, sin re-evaluación por su deceso (Tabla 2) y $22 \mathrm{RN}$ del grupo sin infección congénita.

Dieciocho de los $41 \mathrm{RN}$ excluidos en que no se logró estudio oportuno de CMV eran elegibles para este estudio por resultado "refiere" en la primera etapa del estudio auditivo. Durante el seguimiento de estos pacientes, uno de ellos fue diagnosticado con HSN asimétrica (leve en un oído y moderado en el otro), otro falleció por causa no esclarecida en la ficha clínica, 5 no se presentan a reevaluación auditiva pese a citaciones, y los 11 restantes resultaron normoacúsicos en la segunda etapa del estudio auditivo.

\section{Discusión}

La infección por CMVc sigue siendo un problema de salud pública mundial, debido al impacto que tiene en la audición y en el desarrollo neurocognitivo a largo plazo. $\mathrm{Su}$ pesquisa precoz, permite realizar una intervención terapéutica y el seguimiento adecuado de estos pacientes ${ }^{27}$. En nuestro estudio, cuatro de cinco de los pacientes con CMVc fueron asintomáticos, proporción similar a lo reportado en la literatura médica ${ }^{28}$. La prevalencia de
Tabla 3. Prevalencia de CMVc por grupo de riesgo (se admite más de 1 criterio por RN)

\begin{tabular}{lccc}
\hline Grupo de Riesgo & CMV (+) & Total & Prevalencia CMVc \\
Sospecha activa infección congénita (TORCH) & 1 & 3 & $33,3 \%$ \\
Estudio auditivo alterado (PEATa o EOA) & 2 & 24 & $8,3 \%$ \\
Hijos de madre VIH & 2 & 41 & $4,9 \%$ \\
PEG severo & 3 & 90 & $3,3 \%$ \\
Peso nacimiento < $1.500 \mathrm{~g}$ & 1 & 60 & $1,7 \%$ \\
\hline Edad gestacional $<32 \mathrm{sem}$ & 0 & 65 & $0 \%$ \\
\hline
\end{tabular}

CMVc: citomegalovirus congénito, RN: recién nacido, TORCH: infección congénita, PEATa: potenciales evocados auditivos de tronco automatizado, EOA: emisiones otoacústicas, VIH: virus de inmunodeficiencia humana, PEG: pequeño para la edad gestacional. No se encontraron diferencias estadísticamente significativas entre los grupos.

infección por CMVc en $\mathrm{RN}$ de alto riesgo, en nuestra serie, fue de 2,6\%; valor por sobre lo comunicado previamente en Chile en población de $\mathrm{RN}^{14}$.

Se debe sospechar activamente la infección por CMVc en los RN hijos de madre con antecedente de primoinfección o infección no primaria por CMV durante el embarazo, con alteraciones ecográficas compatibles y en los $\mathrm{RN}$ con manifestaciones clínicas de infección congénita al nacimiento ${ }^{12,14}$. El subgrupo donde se encontró la mayor incidencia en nuestro estudio fue en los pacientes que presentaron alguna alteración en la ecografía prenatal o síntomas sugerentes al nacer (1 de 3 pacientes), siguiendo en frecuencia los RN que "refieren" en el estudio auditivo al nacer $(8,3 \%)$, los hijos de madre con infección por VIH $(4,9 \%)$ y los PEG severos (3,3\%); no obstante, su relación con CMVc no fue estadísticamente significativa.

El cribado de CMV en RN que "refieren" en el estudio auditivo neonatal ("targeted screening") se ha planteado previamente por otros grupos de investigadores. Stehel y cols. ${ }^{29}$, determinaron que $6 \%$ de los RN que "refieren" en estudio auditivo tienen infección por CMVc y que $75 \%$ de ellos fueron identificados solamente por dicho resultado. Es así como en algunas ciudades o estados de Estados Unidos de América, Europa y Australia, se ha implementado el estudio de CMV en distintas muestras (saliva, orina o, en forma retrospectiva, en muestras de papel filtro del cribado neonatal) en todo RN que "refiere" el estudio auditivo al nacer, con resultados variables (incidencias de CMVc entre 1,8 y $6 \%)^{11,28-32}$ y con la limitación de que con esta estrategia no se identifican hasta $43 \%$ de los niños que desarrollan hipoacusia tardía relacionada a $\mathrm{CMV}^{33}$.

Fowler y cols. ${ }^{33}$, en el estudio CHIMES de cribado universal multicéntrico de $\mathrm{CMVc}$, describen que en el grupo de RN con CMVc, 7\% "refiere" en la evaluación auditiva, mientras que en los RN que no presentan infección congénita fue de $0,9 \%(\mathrm{p}<0,0001)$. Asimismo, al 
analizar el grupo de RN hospitalizados en UCIN, se vio que $20 \%$ de los CMVc "refieren" en el estudio auditivo vs $5 \%$ de los RN sin infección por CMVc $(\mathrm{p}<0,001)$.

En nuestra serie, se detectó una incidencia de $8,3 \%$ (2/24) de infección CMVc en pacientes que "refieren" en el estudio auditivo al nacer. No se ha diagnosticado hipoacusia en los cuatro niños con CMVc en seguimiento hasta el año de vida; sin embargo, se debe mantener las evaluaciones auditivas al menos hasta los seis años, ya que 6 a $15 \%{ }^{1,2}$ de los RN con infección por CMVc asintomáticos pueden desarrollar hipoacusia en el tiempo. En nuestro protocolo de estudio se realiza control auditivo trimestral durante el primer año de vida, luego semestral durante el segundo año y luego anual hasta los seis años ${ }^{2,9}$.

Es conocido que, tanto la primoinfección como la infección no primaria por CMV en la mujer embarazada ${ }^{34}$, puede resultar en la transmisión materno-fetal de CMV, con frecuencias de 30 a $40 \%$ y 1 a $2 \%$, respectivamente ${ }^{8}$; siendo más grave y con mayor proporción de secuelas a largo plazo la que ocurre durante el primer trimestre del embarazo (antes de las 14 semanas) ${ }^{35}$. En países con alta seroprevalencia como Chile, donde aproximadamente $90-95 \%$ de las mujeres embarazadas son seropositivas ${ }^{36}$, la principal fuente de infección del RN es la reactivación/ reinfección por CMV en la madre ${ }^{8,37,38}$, como lo demuestra Blasquez-Gamero y cols. ${ }^{39}$, quienes reportaron $71,4 \%$ de $\mathrm{RN}$ con $\mathrm{CMVc}$ infectados por esta vía.

La mujer gestante portadora de VIH tiene mayor riesgo de infección no primaria de CMV durante el embarazo por lo que el hijo de madre con esta infección se ha considerado como de riesgo para la infección congénita por CMV. La prevalencia en nuestro estudio fue de 4,9\%, que concuerda con lo reportado en la literatura médica (2$7 \%)^{20,40-42}$, si bien la incidencia ha ido disminuyendo con la terapia anti-retroviral (TARV) efectiva en los últimos años $^{43,44}$. En Chile, se realiza el estudio de CMV en orina en forma rutinaria a todos los hijos de madre portadora de $\mathrm{VIH}^{20}$. En nuestro reporte, se encontraron dos casos de infección congénita por CMV en este grupo de pacientes, ambas asintomáticas, que no se hubiesen pesquisado sin esta estrategia. Un caso presentó co-infección con VIH confirmado (dos RPC positivas para VIH) y su madre el antecedente de mala adherencia a la TARV durante el embarazo. Adachi y cols. ${ }^{47}$, reportaron una frecuencia cuatro veces mayor en los hijos de madre con VIH infectados que en aquellos expuestos no infectados (OR, 4.4; 95\% IC: 2,3-8,2) y seis veces mayor en aquellos con infección por $\mathrm{VIH}$ in útero (RPC para VIH positivas al nacer) (OR 6 , 95\% IC 3-12,1), por lo que se debe tener mayor atención en aquellos RN hijos de madres portadoras del VIH que no recibieron protocolo de prevención de transmisión vertical, hijos de madres diagnosticadas al parto, con carga viral detectable a las 34 semanas de gestación o con mala adherencia o inicio tardío de $\mathrm{TARV}^{46}$.
La infección por CMV es una causa conocida de restricción de crecimiento intrauterina (PEG severo) ${ }^{28,47}$; no obstante, no hubo una diferencia significativa en el PN (2.390 vs $1.850 \mathrm{~g} ; \mathrm{p}=0,11)$ y la proporción de PEG (60 vs $46,5 \% ; p=0,44)$ entre los pacientes con y sin infección por CMVc. En nuestra serie, en dos de los cinco pacientes con infección por CMVc (40\%) el motivo de estudio fue sólo ser PEG severo. Caso 1: PEG severo hijo de madre con pre-eclampsia y caso 3: PEG severo, hijo de madre con antecedente de multi-consumo de drogas. Ambos $\mathrm{RN}$ tenían una causa explicable de PEG, por lo que habitualmente no se habría hecho el diagnóstico de CMVc. Por ello, al menos en los PEG severos, recomendamos el cribado de CMV.

Los prematuros extremos son otro grupo importante a considerar en el cribado de CMV pues, si bien dicho virus causa prematuridad y bajo $\mathrm{PN}$, los prematuros extremos también presentan un mayor riesgo de adquirir la infección de forma postnatal (CMVp) ${ }^{48}$. Josephson C y cols. ${ }^{49}$, reportaron una prevalencia de $6,9 \%$ en 539 prematuros extremos en forma prospectiva hasta los 3 meses de edad, $17 \%$ presentó enfermedad sintomática (como "sepsis like", neumonía, enterocolitis, hepatitis, trombocitopenia, entre otros) o falleció. En nuestra serie, 60 RN clasificaron con el criterio de PN; uno de ellos (1.400 g y PEG) con $\mathrm{CMVc}(1,7 \%)$. El conocer el estado de infección al nacer permitiría realizar un diagnóstico diferencial más certero con CMVc, por lo que se recomienda el cribado en este grupo. Esto cambiaría los tiempos de tratamiento y el seguimiento de estos pacientes, ya que aún no está claro si la infección por CMVp está asociada a HSN o alteraciones del neurodesarrollo a largo plazo a diferencia del $\mathrm{CMVc}^{50}$.

El diagnóstico de CMVc a través de RPC en muestra de orina tiene una alta sensibilidad y especificidad, y es el estándar de oro a la fecha. Requiere del uso de recolectores de orina, que en $\mathrm{RN}$ de muy bajo PN puede ser complejo debido al contacto que requiere con la frágil piel de estos RN y, además, se debe esperar hasta la micción espontánea del RN. Una alternativa más sencilla podría ser el uso de RPC en hisopado de saliva, donde la obtención de la muestra es inmediata y sin mayores efectos adversos para el RN. Con muestra de saliva, también se mantiene una alta sensibilidad (97,4-100\%) y especificidad $(99,9 \%)^{2,9,51,52}$; sin embargo, requiere de confirmación de los casos positivos con RPC en muestra de orina dado que existe riesgo de contaminación de la muestra luego de la lactancia materna. El estudio con muestra de saliva no fue posible en nuestra realidad local, pero es una herramienta útil y menos invasiva. Otra alternativa usada es el estudio retrospectivo de CMV en las muestras tomadas para el cribado neonatal en papel filtro; sin embargo, éstas mostraron una sensibilidad menor al $50 \%$ en estudios a gran escala ${ }^{53-55}$ y se debe considerar 
prevalencia de la enfermedad y el tamaño de la muestra, no se encontraron diferencias significativas.

En suma, un programa de cribado universal de infección por CMVc permitiría evaluar oportunamente al $\mathrm{RN}$, considerar el tratamiento y realizar un seguimiento activo a largo plazo, agregados a potenciales beneficios sociales y en salud pública por disminución de carga y costo por discapacidad ${ }^{58}$. Los estudios de costo-beneficio realizados, tanto para cribado universal como en grupos de riesgo, han mostrado utilidad ${ }^{59}$; sin embargo, en lugares con recursos limitados, el cribado universal pareciera ser impracticable por ahora, sobre todo porque el tratamiento del RN asintomático sigue siendo controversial. En nuestra realidad se podría recomendar, al menos, el cribado en grupos de riesgo: $\mathrm{RN}$ que refieren el estudio auditivo al nacer, hijos de madre con infección por VIH, prematuros extremos, PEG severos y en todo aquel que se sospecha por antecedentes prenatales o manifestaciones clínicas al nacer. Se necesitan más y nuevos estudios de seroprevalencia en las mujeres embarazadas en el país, dado el cambio poblacional con aumento de inmigrantes en los últimos años, y estudios de cribado de mayor escala para tomar decisiones de salud pública. de $41(43,9 \%)$ de los RN elegibles para el estudio por criterio de estudio auditivo con resultado "refiere" no pudieron ser reclutados porque no se realizó estudio de CMV previo al alta o antes de los $21 \mathrm{ddv}$. Esto ocurre principalmente por el alta hospitalaria de los $\mathrm{RN}$ fuera de horario de reclutamiento (por ej.: fines de semana) y la posterior dificultad para la toma de RPC-CMV de forma ambulatoria. El cribado universal evitaría este problema ya que no habría que esperar a tener el resultado auditivo para solicitar el estudio de CMV y a su vez, el análisis en saliva facilitaría la adherencia a la toma de muestra, sobre todo en los RN prematuros críticos. Se requiere de estudios con mayor número de sujetos, ya que, debido a la
Agradecimientos a: Proyectos de Investigación Laboratorios Saval-2017. Fondos utilizados para la compra de reactivos para la determinación de laboratorio de CMV, Haydee Poblete, Laboratorio de Microbiología Hospital Lucio Córdova, Jessica Jorquera Cares, Lorena Castillo Aedo, Marisa Moncada, Secretarias del Servicio de Neonatología Hospital Barros Luco Trudeau, Daniela Martínez, Secretaria del Programa Tamizaje Auditivo Universal Hospital Barros Luco Trudeau y Siboney Muñoz, Secretaria del Servicio de Otorrinolaringología Hospital Barros Luco Trudeau.

\section{Referencias bibliográficas}

1.- De Vries J J C, Vossen A C T M, Kroes A C M, van der Zeijst B A M. Implementing neonatal screening for congenital cytomegalovirus: addressing the deafness of policy makers. Rev Med Virol. 2011; 21: 54-61. doi: 10.1002/ rmv.679.

2.- Williams E J, Kadambari S, Berrington J E, Luck S, Atkinson C, Walter S, et al. Feasibility and acceptability of targeted screening for congenital CMV-related hearing loss. Arch Dis Child Fetal Neonatal Ed. 2014; 99 (3): 230-6. doi:10.1136/archdischild-2013-305276.

3.- Santos D V., Souza M M, Gonçalves S H, Cotta A C, Melo L A, Andrade G M, et al. Congenital cytomegalovirus infection in a neonatal intensive care unit in brazil evaluated by PCR and association with perinatal aspects. Rev Inst
Med Trop Sao Paulo. 2000; 42 (3): 129-32. doi:10.1590/S0036-46652000000300003.

4.- Kadambari S, Luck S, Davis A, Walter S, Agrup C, Atkinson C, et al. Evaluating the feasibility of integrating salivary testing for congenital CMV into the Newborn Hearing Screening Programme in the UK. Eur J Pediatr. 2015; 174 (8): 1117-21. doi:10.1007/s00431-015-2506-8.

5.- Manicklal S, Emery V C, Lazzarotto T, Boppana S B, Gupta R K. The "Silent" global burden of congenital cytomegalovirus. Clin Microbiol Rev. 2013; 26 (1): 86-102. doi:10.1128/CMR.00062-12.

6.- Stagno S, Dworsky M E, Torres J, Mesa T, Hirsh T. Prevalence and importance of congenital cytomegalovirus infection in three different populations. J Pediatr. 1982; 101 (6): 897-900. doi:10.1016/S00223476(82)80006-1.
7.- Lanzieri T M, Dollard S C, Bialek S R, Grosse S D. Systematic review of the birth prevalence of congenital cytomegalovirus infection in developing countries. Int $\mathrm{J}$ Infect Dis. 2014; 22 (May 2013): 44-8. doi:10.1016/j. ijid.2013.12.010.

8.- Leruez-Ville M, Magny J F, Couderc S, Pichon $\mathrm{C}$, Parodi M, Bussières L, et al. Risk factors for congenital CMV infection following primary and non-primary maternal infection. Clin Infect Dis. 2017; 65 (3): 398-404.

9.- Mestas E. Congenital cytomegalovirus. Adv Neonatal Care. 2016; 16 (1): 60-5. doi:10.1097/ ANC.0000000000000242.

10.- Fowler K B, Boppana S B. Congenital cytomegalovirus (CMV) infection and hearing deficit. J Clin Virol. 2006; 35 (2): 226-31. doi:10.1016/j.jcv.2005.09.016.

11.- Diener M L, Zick C D, McVicar S B, Boettger 


\section{— -}

J, Park A H. Outcomes from a hearing-targeted cytomegalovirus screening program. Pediatrics. 2017; 139 (2). doi:10.1542/peds.2016-0789.

12.- Baquero-Artigao F. Consensus document from the Spanish Society of Paediatric Infectious Diseases (SEIP) on the diagnosis and treatment of congenital cytomegalovirus infection. An Pediatr. 2009; 71 (6): 535-47. doi:10.1016/j. anpedi.2009.07.029.

13.- Amir J, Attias J, Pardo J. Treatment of late-onset hearing loss in infants with congenital cytomegalovirus infection. Clin Pediatr (Phila). 2014; 53 (5): 444-8. doi: $10.1177 / 0009922813510204$.

14.- Luchsinger V, Suárez M, Schultz R, Barraza P, Guzmán M, Ferrada L, et al. Incidence of congenital cytomegalovirus infection in newborn infants of different socioeconomic strata. Rev Med Chil. 1996; 124 (4): 403-8. http://europepmc.org/abstract/MED/9110478.

15.- Kaye CI, Committee on Genetics; Accurso F, La Franchi S, Lane PA, Hope N, Sonya P, et al. Newborn screening fact sheets. Pediatrics. 2006; 118 (3). doi:10.1542/peds.2006-1783.

16.- Ronchi A, Shimamura M, Malhotra $P$ S, Sánchez P J. Encouraging postnatal cytomegalovirus (CMV) screening: the time is NOW for universal screening! Expert Rev Anti Infect Ther. 2017; 15 (5): 417-9. doi:10.1080/1 4787210.2017 .1303377$.

17.- Hilditch C, Liersch B, Spurrier N, Callander E J, Cooper C, Keir A K. Does screening for congenital cytomegalovirus at birth improve longer term hearing outcomes? Arch Dis Child. 2018; 103 (10): 988-92. doi:10.1136/ archdischild-2017-314404.

18.- Cannon M J, Griffiths P D, Aston V, Rawlinson W D. Universal newborn screening for congenital C M V infection: What is the evidence of potential benefit? Rev Med Virol. 2014; 24 (5): 291-307. doi:10.1002/rmv. 1790.

19.- Kawada J ichi, Torii Y, Kawano Y, Suzuki M ${ }^{1}$, Yasuko Kamiya Y, Kotani T, et al. Viral load in children with congenital cytomegalovirus infection identified on newborn hearing screening. J Clin Virol. 2015; 65: 41-5. doi:10.1016/j.jcv.2015.01.015.

20.- Ministerio de Salud de Chile. Guía Clínica AUGE: Síndrome de la Inmunodeficiencia Adquirida VIH/SIDA. Serie Guìas Clínicas MINSAL 2013. https://www.minsal.cl/sites/ default/files/files/GPCVIH.pdf.

21.- Botet F, Figueras Aloy J, Álvarez E, de Alba C, Dorronsolo I, Echaniz Urcelay I, et al. Universal cytomegalovirus infection screening in premature newborns less than $1500 \mathrm{~g}$. An Pediatr. 2014; 81 (4): 256.e1-256.e4. doi:10.1016/j.anpedi.2014.01.011.

22.- Sánchez Pintos J, Figueras Nadal C, Salcedo Abizanda S. Revisión prospectiva de la infección congénita y perinatal por citomegalovirus en pretérminos. En: XXI
Congreso Nacional de Medicina Perinatal (SEN), IV Congreso Iberoamericano de Neonatología (SIBEN) y II Congreso de la SEMP. An Pediatric (Barc) 2007; 67 (Supl 1): 51-204. Pág. 63, Poster 33.

23.- Stagno S, Britt B, Remington J, Klein J. Cytomegalovirus. In: Remington \& Klein. Infectious Diseases of the Fetus and Newborn Infant, $6^{\text {th }}$ ed. Remington J S, Klein J O, Wilson C B, Baker C J. Elsevier, Amsterdam 2006; 1313: 740-81.

24.- Escosa-García L, Baquero-Artigao F, Noguera Julian A, Blázquez Gamero D. Cytomegalovirus screening in less than 1500 g premature newborns. National Congenital Cytomegalovirus Disease Registry Scientific Committee. An Pediatr. 2015; 83 (1): 70-1. doi:10.1016/j.anpedi.2015.02.019.

25.- Ministerio de Salud Chile. Diario Oficial de la República de Chile. Modifica resolución exenta $\mathrm{N}^{\circ} 277 / 2011$ del Minsiterio de Salud. Resolución 54 - punto I. 34 sobre Prestaciones Tamizaje Auditivo. Consulta: https://www.diariooficial.interior.gob.cl/ publicaciones/2020/02/17/42581/01/1727453. pdf.

26.- Joint Committe on Infant Hearing. Year 2007 Position Statement: Principles and guidelines for early hearing detection and intervention programs joint committee on infant hearing. Pediatrics. 2007; 120 (4): 898-921. doi:10.1044/policy.ps2007-00281.

27.- Grosse S D, Dollard S C, Kimberlin D W. Screening for congenital cytomegalovirus after newborn hearing screening: What comes next? Pediatrics. 2017; 139 (2). e20163837. doi:10.1542/peds.2016-3837.

28.- Rawlinson W D, Boppana S B, Fowler K B, Kimberlin D W, Lazzarotto T, Alain S, et al. Congenital cytomegalovirus infection in pregnancy and the neonate: consensus recommendations for prevention, diagnosis, and therapy. Lancet Infect Dis. 2017; 17 (6): e177-e188. doi:10.1016/S1473-3099(17)301433.

29.- Stehel E K, Shoup A G, Owen K E, Jackson G L, Sendelbach D M, Boney L F, et al. Newborn hearing screening and detection of congenital cytomegalovirus infection. Pediatrics. 2008; 121 (5): 970-5. doi:10.1542/peds.2006-3441

30.- Vancor E, Shapiro E D, Loyal J. Results of a targeted screening program for congenital cytomegalovirus infection in infants who fail newborn hearing screening. J Pediatric Infect Dis Soc. 2019; 8 (1): 55-9. doi:10.1093/jpids/ pix 105.

31.- Beswick R, David M, Higashi H, Thomas D, Nourse C, Koh G, et al. Integration of congenital cytomegalovirus screening within a newborn hearing screening programme. $\mathrm{J}$ Paediatr Child Health. 2019; 55 (11): 1381-8. doi:10.1111/jpc. 14428 .
32.- Rawlinson W D, Palasanthiran P, Hall B, Al Yazidi L, Cannon M J, Cottier C, et al. Neonates with congenital cytomegalovirus and hearing loss identified via the universal newborn hearing screening program. J Clin Virol. 2018; 102: 110-5. doi:10.1016/j. jev.2018.03.006.

33.- Fowler K B, McCollister F P, Sabo D L, Shoup A G, Owen K E, Woodruff J L, et al. A targeted approach for congenital cytomegalovirus screening within newborn hearing screening. Pediatrics. 2017; 139 (2). doi:10.1542/ peds.2016-2128.

34.- Cannon M J, Schmid D S, Hyde T B. Review of cytomegalovirus seroprevalence and demographic characteristics associated with infection. Rev Med Virol. 2010; 20 (4): 202-13. doi:10.1002/rmv.655.

35.- Faure-Bardon V, Magny J F, Parodi M, Couderc S, Garcia P, Maillotte A M, et al. Sequelae of congenital cytomegalovirus following maternal primary infections are limited to those acquired in the first trimester of pregnancy. Clin Infect Dis. 2019; 69 (9): 1526-32. doi:10.1093/cid/ ciy1128.

36.- Yamamoto M, Prado P, Wilhelm J, Bradford R, Lira F, Insunza A. et al. Alta prevalencia de IgG anti citomegalovirus en 583 embarazos: Hospital Padre Hurtado. Rev Chil Obstet Ginecol. 2009; 74 (2): 102-6. http://dx.doi. org/10.4067/S0717-75262009000200006.

37.- Yamamoto A Y, Mussi-Pinhata M M, Boppana S B, de Frizzo Oliveira P, Duarte G, Britt WJ, et al. Human cytomegalovirus reinfection is associated with intrauterine transmission in a highly cytomegalovirus-immune maternal population. Am J Obstet Gynecol. 2010; 202 (3): 297.e1-297.e8. doi:10.1016/j. ajog.2009.11.018.

38.- Mussi-Pinhata M M, Yamamoto A Y, Brito R M M, de Lima Isaac M, de Carvalhoe Oliveira P F, Boppana $\mathrm{S}$, et al. Birth prevalence and natural history of congenital cytomegalovirus infection in a highly seroimmune population. Clin Infect Dis. 2009; 49 (4): 522-8. doi:10.1086/600882.

39.- Blázquez-Gamero D, Soriano-Ramos M, Vicente M, Pallás-Alonso C R, Pérez-Rivilla A, García-Álvarez M, et al. Prevalence and clinical manifestations of congenital cytomegalovirus infection in a screening program in Madrid (PICCSA Study). Pediatr Infect Dis J. 2020; 39 (11): 1050-6. doi:10.1097/ inf.0000000000002808.

40.- Mussi-Pinhata M M, Yamamoto A Y, Figueiredo L T M, Cervi M C, Duarte G. Congenital and perinatal cytomegalovirus infection in infants born to mothers infected with human immunodeficiency virus. J Pediatr. 1998; 132 (2): 285-90. doi:10.1016/S00223476(98)70446-9.

41.- Kovacs A, Schluchter M, Easley K, Demmler G, Shearer W, La Russa P, et al. 
Cytomegalovirus infection and HIV-1 disease progression in infants born to HIV-1 infected women. N Engl J Med. 1999; 341 (2): 77-84. doi: 10.1056/NEJM199907083410203.

42.- Doyle M, Atkins J, Rivera-Matos I. Congenital cytomegalovirus infection in infants infected with human immunodeficiency virus type 1 . Pediatr Infect Dis J. 1996; 15 (12): 1102-6. doi: 10.1097/00006454-199612000-00010.

43.- Purswani M U, Russell J S, Dietrich M, Malee K, Spector S A, Williams P L, et al. Birth prevalence of congenital cytomegalovirus infection in HIV-exposed uninfected children in the era of combination antiretroviral therapy. J Pediatr. 2020; 216: 82-7.e2. doi:10.1016/j. jpeds.2019.09.025.

44.- Guibert G, Warszawski J, Le Chenadec J, Blanche S, Benmebarek Y, Mandelbrot L, et al. Decreased risk of congenital cytomegalovirus infection in children born to HIV-1-infected mothers in the era of highly active antiretroviral therapy. Clin Infect Dis. 2009; 48 (11): 151625. doi:10.1086/598934.

45.- Adachi K, Xu J, Ank B, Watts D H, Camarca M, Mofenson L M, et al. Congenital cytomegalovirus and HIV perinatal transmission. Pediatr Infect Dis J. 2018; 37 (10): 1016-21. doi:10.1097/ INF.0000000000001975.

46.- Ministerio de Salud Chile Programa Nacional de Prevención y Control de la infección por VIH/SIDA e ITS. Norma Conjunta de Prevención de La Transmisión Vertical Del VIH y La Sífilis 2013. http://www.scielo.cl/pdf/rci/ v30n3/art04.pdf.

47.- Cofre F, Delpiano L, Labraña Y, Reyes A, Sandoval A, Izquierdo G. Síndrome de TORCH: enfoque racional del diagnóstico y tratamiento pre y post natal. Recomendaciones del Comité Consultivo de Infecciones Neonatales Sociedad Chilena de Infectología, 2016. Rev Chil infectología. 2016; 33 (2): 191216. doi:10.4067/s0716-10182016000200010

48.- Kadambari S, Luck S, Heath P T, Sharland M. Preemptive screening strategies to identify postnatal CMV diseases on the neonatal unit. Pediatr Infect Dis J. 2016; 35 (10): 1148-50. doi:10.1097/INF.0000000000001303.

49.- Josephson C D, Caliendo A M, Easley K A, Knezevic A, Shenvi N, Hinkes M T, et al. Blood transfusion and breast milk transmission of cytomegalovirus in very low-birth-weight infants: A prospective cohort study. JAMA Pediatr. 2014; 168 (11): 1054-62. doi:10.1001/ jamapediatrics.2014.1360.

50.- Wright C J, Permar S R. Preventing postnatal cytomegalovirus infection in the preterm infant: Should it be done, can it be done, and at what cost? J Pediatr. 2015; 166 (4): 795-8. doi:10.1016/j.jpeds.2014.12.062.

51.- Kadambari S, Williams E J, Luck S, Griffiths P D, Sharland M. Evidence based management guidelines for the detection and treatment of congenital CMV. Early Hum Dev. 2011; 87 (11): 723-8. doi:10.1016/j. earlhumdev.2011.08.021.

52.- Boppana S B, Ross S A, Shimamura M, Palmer A L, Ahmed A, Michaels M G, et al. Saliva polymerase-chain-reaction assay for cytomegalovirus screening in newborns. N Engl J Med. 2011; 364 (22): 2111-8. doi:10.1056/ NEJMoa1006561.

53.- Lüsebrink N, Kieslich M, Rabenau H F, Schlößer R L, Buxmann H. Retrospectively diagnosing congenital cytomegalovirus infections in symptomatic infants is challenging. Acta Paediatr 2021 Jan; 110 (1):197-202. doi:10.1111/apa.15305.
54.- Boppana S B, Ross S A, Novak Z, Shimamura M, Tolan R Jr, Palmer A L, et al. Dried blood spot real-time polymerase chain reaction assays to screen newborns for congenital cytomegalovirus infection. Obstet Gynecol Surv. 2010; 65 (8): 484-5. doi:10.1097/ OGX.0b013e3181f0795b.

55.- Ross S A, Ahmed A, Palmer A L, Fowler K B, Boppana S B, for the C M V and Hearing Multicenter Screening (CHIMES) Study Group. Newborn dried blood spot polymerase chain reaction to identify infants with congenital cytomegalovirus-associated sensorineural hearing loss. J Pediatr. 2017; 184: 57-61.e1. doi:10.1016/j.jpeds.2017.01.047.

56.- Kimberlin D W, Acosta E P, Sánchez P J, Sood $\mathrm{S}$, Agrawal V, Homans J, et al. Pharmacokinetic and pharmacodynamic assessment of oral valganciclovir in the treatment of symptomatic congenital cytomegalovirus disease. J Infect Dis. 2008; 197 (6): 836-45. doi:10.1086/528376.

57.- Walter S, Atkinson C, Sharland M, Rice P, Raglan E, Emery V C ,et al. Congenital cytomegalovirus: association between dried blood spot viral load and hearing loss. Arch Dis Child Fetal Neonatal Ed. 2008; 93 (4): F280-5. doi:10.1136/adc.2007.119230.

58.- Lazzarotto T, Blázquez-Gamero D, Delforge M $\mathrm{L}$, et al. Congenital cytomegalovirus infection: a narrative review of the issues in screening and management from a panel of European experts. Front Pediatr. 2020; 8: 13. doi:10.3389/ fped.2020.00013.

59.- Lunardi S, Lorenzoni F, Ghirri P. Universal screening for congenital CMV infection. Update Crit Issues Infant Neonatal Care. Published online 2020; 1-14. doi:10.5772/ intechopen.89611. 